\title{
Klinische Studien in der Onkologie - 2013 und zukünftig
}

\author{
Bernhard Wörmann $^{\mathrm{a}, \mathrm{b}} \quad$ Mathias Freund ${ }^{\mathrm{c}} \quad$ Friedrich Overkamp $^{\mathrm{d}} \quad$ Gerhard Ehninger $^{\mathrm{e}}$ \\ ${ }^{\text {a }}$ Deutsche Gesellschaft für Hämatologie und Medizinische Onkologie, \\ ${ }^{\mathrm{b}}$ Ambulantes Gesundheitszentrum der Charité Universitätsmedizin Berlin, Medizinische Klinik mit Schwerpunkt Hämatologie \\ und Onkologie, Campus Virchow Klinikum, Berlin, \\ ${ }^{\mathrm{c}}$ Rostock, \\ ${ }^{\text {d }}$ Praxis und Tagesklinik für Internistische Onkologie und Hämatologie, Recklinghausen, \\ ${ }^{e}$ Medizinische Klinik und Poliklinik I, Universitätsklinikum Carl Gustav Carus an der Technischen Universität Dresden, \\ Dresden, Deutschland
}

\section{Schlüsselwörter \\ Klinische Studien · Onkologie . \\ Nutzenbewertung · Arzneimittelgesetz}

\section{Zusammenfassung}

Klinische Studien sind die Brücke zwischen Grundlagenforschung und Patientenversorgung. Sie sind die Basis der evidenzbasierten Medizin, das Rückgrat von Leitlinien. Die rasche Umsetzung von Erkenntnissen onkologischer Forschung in den letzten 40 Jahren war nur durch eine Vielfalt und einen Organisationsgrad klinischer Studien möglich, wie sie es in kaum einem anderen Gebiet der Medizin gibt. Aktuell ist diese Studienkultur in Deutschland gefährdet. Tiefgreifende Änderungen der politischen Rahmenbedingungen, unzureichende Finanzierung, neue Definition patientenrelevanter Endpunkte und veränderte Strukturen im ambulanten und stationären Bereich erfordern kritische Reflektion und neue Konzepte.

\section{Einleitung}

Die rasante Entwicklung der Onkologie ist eng mit der Durchführung klinischer Studien verbunden. Das strukturierte Vorgehen von Phase I bis Phase IV schafft transparente Regeln für die Evaluation neuer Substanzen und Verfahren und berücksichtigt die ethischen Anforderungen des maximalen Patientenschutzes.

\author{
Keywords \\ Clinical trials - Medical oncology · \\ Risk-benefit assessment · Legislation
}

\section{Summary}

Status of Clinical Trials in Oncology - 2013 and Onwards Clinical trials connect basic science with patient care. They form the backbone of evidence-based medicine and clinical guidelines. The unprecedented implementation of new methods in oncology over the past 40 years has only been possible on the basis of multiple well-organized clinical study groups. The continued existence of these study groups in their current multitude is in danger. Far-reaching changes in the legal framework, underfunding, new definitions of patient-related outcome and shifts in the organization of cancer patient care ask for critical reappraisal and new concepts.

\section{KARGER \\ Fax +497614520714 \\ Information@Karger.com}

www.karger.com

\section{(c) 2013 S. Karger GmbH, Freiburg}

0378-584X/13/3614-0003\$38.00/0

Accessible online at:

www.karger.com/onk
Voraussetzung für die Etablierung eines neuen Therapieverfahrens sind heute fast immer Ergebnisse von einer oder mehreren Phase-III-Studien. In diesem Vorgehen haben auch Studien mit negativem Endergebnis eine nachhaltige Funktion: Multizentrische Studiengruppen definieren zu Beginn einer Studie den derzeit gültigen Vergleichsarm in oft komplexen Krankheitssituationen und legen damit diagnostische und therapeutische Standards fest. 
Die deutsche Hämatologie und Onkologie hatte die Notwendigkeit klinischer Studien in der 2. Hälfte der 1970erJahre erkannt und durch die Gründung multizentrischer Studiengruppen weltweit Anerkennung erworben.

In den letzten Jahren haben sich die Rahmenbedingungen für die Durchführung klinischer Studien verändert, und vor allem für Therapieoptimierungsstudien in der Hämatologie und Onkologie haben sie sich verschlechtert. Exemplarisch sichtbar ist die Krise in der pädiatrischen Hämatologie und Onkologie mit einem signifikanten Rückgang in der Zahl von Studien und Studienpatienten.

Eine Krise ist Anlass zur Reflektion über zu konservierendes und über Reaktionen auf neue Herausforderungen. In diesem Sonderheft werden einige aktuelle Aspekte der Diskussion zu klinischen Studien dargestellt, in detaillierter Weise und zum Teil auch in provokativer Form. Diese Einführung ist auch ein Lotse durch die verschiedenen Artikel dieses Sonderheftes.

\section{Geschichte klinischer Studien in der Onkologie}

Die ersten randomisierten Studien in der Onkologie waren Medikamentenstudien. Das methodische Konzept und die Systematik bauten auf den langsam gewachsenen Erfahrungen der vorangegangenen 200 Jahre auf [1]. Als erstes Beispiel einer vergleichenden Studie gilt die vom schottischen Schiffsarzt James Lind durchgeführte und publizierte Untersuchung über die Behandlung des Skorbut (z.B. Lind [2]). Zu Beginn des 19. Jahrhunderts erschienen Arbeiten über die Notwendigkeit einer sorgfältigen statistischen Auswertung von Behandlungserfolgen. Regeln wurden aufgestellt und unter anderem von Pierre Charles Alexandre Louis im Jahr 1835 bei seiner Untersuchung zum Sinn des Aderlasses bei Patienten mit Pneumonie und Infekten der oberen Luftwege angewandt.

Die grundlegenden Konzepte über adäquate Vergleichsgruppen und die Vermeidung eines Untersucherbias wurden seit der 2. Hälfte des 19. Jahrhunderts vor allem in der Infektiologie entwickelt [3]. Als Vergleich dienten zunächst historische Kontrollen, z.B. von Joseph Lister in der 1870 in The Lancet publizierten Studie über die von ihm entwickelten Regeln zur Vermeidung der Sepsis. Auch Emil von Behring verglich die Wirksamkeit eines von ihm mitentwickelten, gegen das Diphtherietoxin gerichteten Pferdeserums zunächst mit dem Verlauf von Patienten, die am selben Hospital im vorausgegangenen Jahr behandelt worden waren. Zur Überprüfung dieses Ergebnisses an einem größeren Patientenkollektiv führte Johannes A.G. Fibiger in Dänemark die erste große klinische Studie mit einer Zufallsentscheidung («Randomisierung») zwischen den Therapiearmen durch.

Eine Weiterentwicklung in der Methodik zur Effektivitätstestung von Pharmaka im Rahmen von klinischen Studien war die Einführung einer unwirksamen, indifferenten Medikation («Placebo») im Kontrollarm der Studie. Fergus R.
Tab. 1. Zugelassene Substanzen in der Onkologie (Status November 2012)

\begin{tabular}{llc}
\hline Substanzgruppe & Zugelassen & Neu seit 2005 \\
\hline Zytostatika & 62 & 10 \\
Antihormonpräparate & 22 & 3 \\
Gezielte Medikamente & 20 & 14 \\
Monoklonale Antikörper & 10 & 6 \\
Immuntherapie & 7 & 2 \\
\hline
\end{tabular}

Ferguson und Mitarbeiter testeten die Effektivität von Grippeimpfungen durch Injektion von entweder Impfstoff oder Kochsalz. Randomisierung jedes zweiten aufgenommenen Patienten war das gängige Prinzip bei der Prüfung neuer antimikrobiell wirksamer Substanzen wie den Sulfonamiden, Penicillin und Antimalariamitteln. Als Beginn der Ära moderner klinischer Studien gilt die 1948 vom Medical Research Council in Großbritannien organisierte, randomisierte Doppelblindstudie zur Therapie der Tuberkulose mit Streptomycin. Interessanterweise hatte sie einen methodisch ähnlich aufgebauten und bereits 1944 publizierten Vorläufer über die Wirkung des unter anderem in faulenden Äpfeln enthaltenden Mycotoxin Patulin als Medikament gegen Erkältungskrankheiten. 1348 Patienten wurden randomisiert, das Ergebnis war aber negativ, und die Studie wurde auch in der Geschichte klinischer Studien (fast) vergessen [4].

Die ersten randomisierten Studien bei Tumorpatienten wurden seit 1954 in der hämatologischen Onkologie geplant [5] und 1958 erstmals von Emil Frei et al. in Blood mit Vergleich einer intermittierenden versus einer kontinuierlichen Gabe von Methotrexat bei 65 Patienten mit akuter Leukämie publiziert [6].

Ein weiterer Meilenstein war der Nachweis der Effektivität von Chemotherapie in Remission in einer 1963 publizierten Cancer and Leukemia Group B(CALGB)-Studie mit dem paarweisen Vergleich von Mercaptopurin versus Placebo bei Kindern mit akuter Leukämie. Konzeptionell war diese Untersuchung der Vorläufer für die großen Studien zum Wert einer adjuvanten Chemotherapie auch bei soliden Tumoren, zuerst gezeigt für das Mammakarzinom 1975 durch Fisher et al. [7] und 1975 mit Einführung des Cyclophosphamid/ Methotrexat/5-Fluoruracil(CMF)-Schemas durch Bonadonna et al. [8].

Heute sind in Deutschland über 120 Substanzen für die antineoplastische Therapie zugelassen (Tab. 1). Die Auflistung zeigt deutlich den Umschwung innerhalb der letzten Jahre von der konventionellen Zytostatikatherapie zu monoklonalen Antikörpern oder anderen, gezielteren Medikamenten. Die Liste belegt auch den weiterhin raschen Zuwachs an wirksamen Substanzen.

Parallel zur Definition von einheitlichen Ein- und Ausschlusskriterien wurden die statistischen Methoden weiterentwickelt. Für den klinisch tätigen Onkologen war dies die Evolution von der Vier-Felder-Tafel zum Hazard Ratio. 


\section{Finanzierung und Organisation}

Die weltweit standardsetzenden Studien der deutschen hämatologischen Onkologie in den 1980er- und 1990er-Jahren waren Therapieoptimierungsstudien und Investigator-initiierte Studien (IIT). Sie wurden seit 1978 vom Bundesministerium für Forschung und Technologie (BMFT) und in Teilen seit 1999 durch das Bundesministerium für Bildung und Forschung (BMBF) im Rahmen der Kompetenznetzwerke «Akute und Chronische Leukämien» bzw. «Maligne Lymphome» gefördert. Unter vielen Gesichtspunkten war das eine günstige Situation. Die unabhängige Finanzierung ermöglichte Studien nicht nur zu einzelnen Substanzen, sondern zu Therapiekonzepten. Sie sicherte die Erhebung der kritisch notwendigen Daten in der Langzeitbeobachtung. Retrospektiv muss aber auch gesagt werden, dass ein nicht unerheblicher Teil der mit der Studie verbundenen Arbeit nicht honoriert, sondern - ausgesprochen oder unausgesprochen - von den Krankenhausträgern getragen oder von den vor Ort tätigen Studienärzten ehrenamtlich geleistet wurde.

Auch heute sind viele Studien unterfinanziert. Im Beitrag von F. Overkamp und A. Bröker in diesem Heft («Das ökonomische Dilemma») wird das Problem thematisiert. Der Artikel macht die hohe Motivation und das Engagement für die Teilnahme an klinischen Studien deutlich, aber auch die kurz- und mittelfristigen Folgen der nicht ausreichenden Finanzierung.

In den Anforderungen für onkologische Zentren, sowohl in der ganzheitlichen (Deutsche Gesellschaft für Hämatologie und Medizinische Onkologie (DGHO)) als auch der primär organbezogenen Form (Deutsche Krebsgesellschaft (DKG) und andere), ist die aktive Teilnahme an klinischen Studien eine unabdingbare Voraussetzung. Dennoch wird die damit verbundene Arbeit personell nicht überall adäquat abgebildet.

In der Europäischen Union (EU) werden heute etwa $60 \%$ aller klinischen Studien in Verantwortung der pharmazeutischen Industrie und etwa 40\% von anderen Interessenträgern durchgeführt. Pharmazeutische Unternehmen sind entscheidend für die Finanzierung von Medikamentenstudien. Das Forschungsbudget einiger der global agierenden Konzerne überschreitet den Etat des BMFT. Durch die Auswahl der zur Prüfung gelangenden Substanzen, die Festlegung der Indikationen, Einschluss- und Ausschlusskriterien, Dosierung und Dauer von Therapie usw. haben diese Studien und damit die pharmazeutische Industrie großen Einfluss auf die direkte Patientenversorgung.

Die aktuell durchgeführte und in diesem Heft präsentierte Umfrage von K. Ihrig et al. («Akademische multizentrische Studien im Fokus: Auswirkungen der Arzneimitttelgesetzgebung auf Therapieoptimierungsstudien in der Hämatologie/ Onkologie») bei Teilnehmern an IIT in Deutschland zeigt die Gefahr, dass die Teilnahme an den nicht von der pharmazeutischen Industrie finanzierten Studien in der nahen Zukunft um weitere $35 \%$ abnehmen kann. Diese Zahlen machen die Notwendigkeit von besseren, öffentlichen Fördermaßnahmen für diesen zentralen Bestandteil der medizinischen Forschung noch einmal sehr deutlich.

Ein anderer Weg ist die unabhängige Überprüfung schon etablierter Untersuchungs- und Behandlungsverfahren. S. Lange und J. Windeler vom Institut für Qualität und Wirtschaftlichkeit im Gesundheitswesen (IQWiG) plädieren in ihrem Artikel («Die Notwendigkeit unabhängiger klinischer Studien aus der Sicht des Instituts für Qualität und Wirtschaftlichkeit im Gesundheitswesen») für bessere und angemessene Fördermöglichkeiten solcher Studien, vor allem auch im Bereich der nichtmedikamentösen therapeutischen Methoden und bei diagnostischen Verfahren. Sie listen 9 Studien auf, die in den letzten 10 Jahren durch Beschlüsse des Gemeinsamen Bundesausschusses (G-BA) initiiert wurden. Die Liste ist leider sehr kurz und lässt zweifeln, ob dieses Instrument zur Initiierung von Studien angenommen wird. Die Studieninhalte wirken eklektisch. Die Liste wird noch kürzer, da nicht jeder der involvierten Studienleiter weiß, dass seine Studie als vom G-BA initiiert betrachtet wird (ETAL-1).

Die Neufassung des $\S 137$ e des Fünften Buches des Sozialgesetzbuches (SGB V) ermöglicht dem G-BA, eine Richtlinie zur Erprobung des Nutzens einer neuen Methode zu beschließen. Die betroffene Untersuchungs- oder Behandlungsmethode wird in einem befristeten Zeitraum im Rahmen der Krankenbehandlung oder der Früherkennung zulasten der Krankenkassen erbracht. Hier werden erstmals auch in Deutschland die Krankenkassen direkt in die Finanzierung klinischer Studien einbezogen. Ob sie diesen Gestaltungsraum wahrnehmen und ob dieses Konstrukt geeignet ist, die Lücke in der Finanzierung unabhängiger Studien zu schließen, ist zurzeit nicht absehbar.

\section{Gesetzliche Rahmenbedingungen}

\section{Arzneimittelgesetz-Novelle von 2004}

Einen wesentlichen Einfluss auf die Durchführung klinischer Studien hatte die 12. Arzneimittelgesetz(AMG)-Novelle von 2004. Sie setzte die Vorgaben der europäischen Richtlinie 2001/20/EG in deutsches Recht um. Ziel war eine Homogenisierung der zum Teil sehr unterschiedlichen Studienbedingungen in den europäischen Mitgliedsstaaten. Durch die AMGNovelle wurden IIT und Therapieoptimierungsstudien den Zulassungsstudien für Medikamente gleichgesetzt.

Die Zahl der Anträge für klinische Prüfungen ging von 2007 bis 2011 um 25\% zurück. Gleichzeitig stiegen die Kosten. Die EU hat errechnet, dass heute doppelt so viel Personal für die Bearbeitung des Verfahrens zur Genehmigung einer klinischen Studie benötigt wird, konkret eine Steigerung um $107 \%$ bei Studien der pharmazeutischen Industrie, um 98\% bei nichtkommerziellen Sponsoren [9]. Die Versicherungsprämien für Sponsoren aus der Wirtschaft sind seit Einführung der Richtlinie um $80 \%$ gestiegen, die Vorlaufzeit hat sich um $90 \%$ verlängert. 
N. Gökbuget und R. Naumann vom Arbeitskreis Arzneimittelgesetz der DGHO schildern die konkreten Probleme multizentrischer Studiengruppen in ihrem Artikel «Beantragung der Bewilligung von akademischen Multicenterstudien bei deutschen Ethikkommissionen». Die Hindernisse liegen vor allem in den sehr zeitaufwendigen Prozessen der Aktivierung vieler Zentren, in der Beantragung von Ethikvoten, der Vertragslogistik, der Wahrnehmung der Prüfarztpflichten bei Studien in der Versorgungssituation sowie beim Monitoring und in der Überwachung der Studien.

\section{Entwurf einer neuen Verordnung des Europäischen Parlaments und des Rates}

Die Europäische Kommission hat im Juli 2012 einen Vorschlag für eine neue Verordnung über die klinische Prüfung mit $\mathrm{Hu}$ manarzneimitteln vorgelegt. Durch die Verordnung würde die bisher gültige Richtlinie 2001/20/EG aufgehoben [9]. Der jetzt vorgelegte Entwurf beinhaltet wesentliche Änderungen gegenüber der bisherigen Praxis. Die größten Änderungen betreffen die Definition und Regelung des Bereichs «Minimalinterventionelle Klinische Prüfungen» und die Vereinheitlichung der Bewertungsverfahren in den Mitgliedsländern.

Voraussetzungen für die Klassifikation als minimalinterventionelle klinische Prüfung sind die Verwendung von bereits zugelassenen Prüfpräparaten oder von im betroffenen Mitgliedsstaat als Standardbehandlung verwendeten Präparaten und die Anwendung von zusätzlichen diagnostischen oder Überwachungsverfahren mit einem im Vergleich zur normalen klinischen Praxis nur geringfügig zusätzlichen Risiko oder geringfügig zusätzlicher Belastung für den Patienten. Diese Kriterien treffen auf die große Mehrheit der von den multizentrischen Studiengruppen, z.B. innerhalb der Kompetenznetzwerke, initiierten Therapieoptimierungsstudien zu.

Die zweite große Änderung betrifft die Genehmigung von Studien. Es ist ein zweiteiliger Bewertungsbericht vorgesehen. Für den ersten Teil ist der berichterstattende Mitgliedsstaat des Leiters der klinischen Prüfung verantwortlich, für den zweiten die jeweils als Studienteilnehmer betroffenen Staaten. Durch klare Zeitvorgaben und das Prinzip der stillschweigenden Genehmigung kann der Prozess der Bewertung und Genehmigung erheblich beschleunigt werden. Die Festlegung der geeigneten Stelle zur Genehmigung klinischer Studien oder die genaue Arbeitsweise der Ethikkommission wird im Entwurf der EU-Direktive nicht geregelt, sondern als nationale Aufgabe angesehen. Der erreichte hohe ethische Standard wird dabei erhalten und in seiner umfassenden Bedeutung gewürdigt: Wissenschaft und Ethik sind untrennbar. Das Verfahren erlaubt Mitgliedstaaten auch, sich in bestimmten und genau definierten Fällen den Schlussfolgerungen der Bewertung eines Antrags nicht anzuschließen («qualifiziertes Opt-out»).

Die vorgeschlagene Direktive ist ein großer Schritt in die richtige Richtung. Sie erlaubt eine differenzierte Unterteilung von klinischen Studien entsprechend dem Risiko für den Patienten. Die administrativen Vorschläge beschleunigen das Antragsverfahren und berücksichtigen dabei gleichzeitig nationale Standards.

\section{Nutzenbewertung}

Frühe Nutzenbewertung nach dem Arzneimittelneuordnungsgesetz.

Anfang 2011 wurde in Deutschland eine Frühe Nutzenbewertung neu zugelassener Medikamente im Rahmen des Arzneimittelneuordnungsgesetzes (AMNOG) eingeführt [10, 11]. Politisches Ziel war die Begrenzung der Ausgaben für die zahlreichen neuen, in der Regel hochpreisigen Medikamente. Die Frühe Nutzenbewertung durch den G-BA ist die Basis von Preisverhandlungen zwischen den Kostenträgern und den pharmazeutischen Unternehmen.

In der Onkologie wurden bis November 2012 sieben Präparate bewertet (Abirateron (Zytiga ${ }^{\circledR}$ ), Cabazitaxel (Jevtana $^{\circledR}$ ), Eribulin $\left(\right.$ Halaven $\left.^{\circledR}\right)$, Ipilimumab (Yervoy ${ }^{\circledR}$ ), Tegafur/ Gimeracil/Oteracil (Teysuno ${ }^{\circledR}$ ), Vandetanib (Caprelsa ${ }^{\circledR}$ ) und Vemurafenib (Zelboraf $\left.{ }^{\circledR}\right)$ ). Die bisherigen Erfahrungen haben vor allem gezeigt, dass die Kriterien der Nutzenbewertung und die Kriterien der Medikamentenzulassung nicht identisch sind. Eine bei jeder Anhörung intensiv diskutierte Frage ist die Abwägung des Nutzens gegenüber dem Schaden.

Was ist individueller Nutzen: Verlängerung der Überlebenszeit? Verlängerung des progressionsfreien Überlebens? Ansprechraten mit Reduktion der Tumormasse? Symptomlinderung? Verbesserung der Lebensqualität? Der G-BA hat drei positive Bewertungskategorien vorgegeben: «erheblich», «beträchtlich» und «gering». Methodologisch hat das IQWiG diese Kategorien mit Grenzwerten für das relative Risiko und mit Konfidenzintervallen belegt. In den ersten IQWiG-Gutachten wurde die Berechnung des Nutzens ausschließlich auf eine signifikante Verlängerung der Überlebenszeit begrenzt. Als Basis für das Kriterium «erheblich» wurden Grenzwerte einer Auswertung von National Cancer Institute(NCI)-Studien aus dem Jahre 2008 genommen. Danach würden 2\% aller Studien in die höchste Kategorie fallen.

Was ist Schaden? Das IQWiG verwendete eine (additive) Berechnung von unerwünschten Ereignissen nach den CTCAEKriterien (allgemeine Terminologiekriterien von unerwünschten Ereignissen). Diese CTCAE-Kriterien erlauben zwar eine standardisierte Einordnung von über 1200 Symptomen und Laborbefunden, aber keine Bewertung. Intuitiv versteht jeder, dass eine transiente Transaminasenerhöhung CTCAE Grad 3 nicht dieselbe Bedeutung wie z.B. eine vielleicht irreversible Neuropathie des formal selben Schweregrades hat. In einem mathematischen Modell ist das schwer abbildbar.

In der pharmazeutischen Industrie sind die Methoden der Nutzenbewertung von größter Bedeutung für die zukünftige Planung klinischer Studien. Die Initiierung finanziell aufwen- 
diger Phase-III-Studien wird auch davon abhängig gemacht, ob die Kriterien einer Nutzenbewertung erfüllt und damit für die Shareholder interessante Gewinnmargen werden erzielen können.

Die ursprünglich als Instrument der Kostenreduktion gestartete Frühe Nutzenbewertung setzt jetzt Normen für zukünftige Medikamentenstudien.

\section{Patientenbezogene Endpunkte}

Auch international gibt es eine umfassende Diskussion über patientenbezogene Endpunkte (patient-related outcome) in klinischen Studien. Anlass ist vor allem die zunehmende Bedeutung von ökonomischen Bewertungen im Rahmen von Health Technology Assessment(HTA)-Projekten.

Ausgangspunkt ist eine Entwicklung der letzten Jahre, nach der nur eine signifikante Verlängerung der Gesamtüberlebenszeit als primärer Endpunkt von Zulassungsbehörden und anderen akzeptiert wurde. Auch die Bewertung ökonomischen Nutzens ist einfacher durchführbar mit nur einem, zuverlässig messbaren Endpunkt. Was biomathematisch nachvollziehbar ist («nur ein toter Patient ist ein gut dokumentierter Patient»), klingt für Betroffene und behandelnde Ärzte zynisch.

Unter klinischen und ethischen Gesichtspunkten ist der Parameter Gesamtüberlebenszeit als dominierender Endpunkt problematisch. Bei Erkrankungen mit hoher Heilungsrate oder bei Erkrankungen mit langem chronischem Verlauf kann es viele Jahre dauern, bis eine valide Auswertung der Überlebenszeit möglich ist. Wenn Überlebenszeit bei allen Erkrankungen der einzig akzeptierte Parameter wäre, hätte Imatinib heute noch keine Zulassung bei der chronischen myeloischen Leukämie (CML). Dazu kommt, dass eindeutig wirksame Substanzen den Patienten der Vergleichsgruppe bei progredienter Erkrankung nicht vorenthalten werden können (Crossover). Ein aktuelles Beispiel ist die Zulassungsstudie für Vandetanib bei Patienten mit medullärem Schilddrüsenkarzinom. 12 von 13 Remissionen im Kontrollarm wurden durch Gabe von Vandetanib in der anschließenden «OpenLabel-Phase» erzielt. In der Folge dieses ethisch begründeten Studiendesigns ist eine biomathematisch «reine» Auswertung der Gesamtüberlebenszeit nicht möglich. Neue rechnerische Ansätze wie «Inverse Probability of Censoring Weighted Analysis» [12] erlauben retrospektive Berechnungen des Einflusses eines Crossovers, sind aber in ihrer Anwendbarkeit noch umstritten.

Das krankheits- bzw. progressionsfreie Überleben ist bei zahlreichen Erkrankungssituationen ein geeigneter Surrogatmarker. Die Messung der Progression muss krankheitsspezifisch erfolgen. Das kann bei einer Substanz wie Catamuxomab die Zunahme der Frequenz von Aszitespunktionen, bei neuen Tyrosinkinase-Inhibitoren bei der CML das molekulare Rezidiv sein.

Ein komplexes Thema ist die Bewertung des individuellen Nutzens einer Therapie. Intuitiv ist dies der Parameter mit der höchsten Wertigkeit, weil er der täglich erlebten Situation des Shared Decision Making im Arzt-PatientenGespräch entspricht. Der potenzielle Nutzen einer Therapie ist von zahlreichen, durch die persönliche Lebenssituation des Patienten bestimmten Faktoren abhängig. Neuere Modelle bilden diese komplexe Abwägung des individuell gesetzten Therapieziels (Heilung, längere Überlebenszeit, längere Zeit bis zum Progress, Symptomfreiheit oder -linderung) gegenüber den unerwünschten Wirkungen (objektiv messbare Nebenwirkungen, subjektive Nebenwirkungen, Abhängigkeit und andere) besser $\mathrm{ab}$ und werden in prospektiven Studien auf ihre Reproduzierbarkeit und ihre Aussagekraft getestet.

\section{Ethik und Ethikkommissionen}

Der Durchführung klinischer Studien liegt ein ethischer Konflikt zugrunde: Das Interesse der Gesellschaft - objektive Überprüfung neuer Verfahren, Beachtung ökonomischer Rahmenbedingungen - ist nicht per se deckungsgleich mit den primären Interessen der betroffenen Patienten - Lebensverlängerung, Symptomlinderung. Der Patient stimmt der Behandlung im Rahmen einer Placebo-kontrollierten klinischen Studie in der Hoffnung zu, nicht das Placebo zu erhalten.

Die formalen, ethischen Voraussetzungen für eine klinische Studie sind heute leichter zu erfüllen. Modellformulare sind von vertrauenswürdigen Internetseiten herunterzuladen, die Kriterien für den Abbruch einer Studie bei signifikanter Unter- oder Überlegenheit sind definiert, Crossover-Design mit dem Recht des Patienten auf die Prüfsubstanz bei Krankheitsprogress ist bei Medikamentenstudien akzeptiert.

Die inhaltlichen, ethischen Voraussetzungen sind weiterhin eine Herausforderung. Dies betrifft zum einen das Design. Patienten im Kontrollarm dürfen nicht suboptimal behandelt werden. Je schlechter das Ergebnis im Kontrollarm, umso höher die Chance für einen signifikanten Vorteil des neuen Medikamentes. Mögliche Beispiele sind die Verwendung von Carboplatin statt Cisplatin im Vergleichsarm von Studien beim nichtkleinzelligen Lungenkarzinom oder von Daunoblastin $45 \mathrm{mg} / \mathrm{m}^{2}$ statt $\geq 60 \mathrm{mg} / \mathrm{m}^{2}$ bei Patienten mit akuter myeloischer Leukämie (AML).

Eine weitere, ungelöste Herausforderung ist die Aufklärung von Patienten. Dies ist nicht durch perfekte Aufklärungsbögen vom Umfang eines Sonderheftes zu lösen. Informed Consent ist eine formalistische Anforderung. Die Sicherstellung, dass die begrenzte Aufnahmefähigkeit des schwer kranken Patienten nicht ausgenützt wird, ist eine Aufgabe des ärztlichen Urteilsvermögens. Bei allem Respekt vor dem in Deutschland erreichten hohen Standard ist weiterhin nicht belegt, dass die Anzahl von begutachtenden Ethikkommissionen linear mit der ethischen Qualität der aktivierten Studie korreliert.

Kontinuierliche Weiterbildung in ethischen Fragen, einschließlich Supervision und Kommunikationstraining, ist auch ein Bestandteil der zukünftigen Studienkultur. 


\section{Langzeitbeobachtung}

Der ökonomische Druck bei der Zulassung von Medikamenten und das neu eingerichtete Instrument der Frühen Nutzenbewertung in Deutschland - 1 Jahr nach der Zulassung - machen klinische Studien kurzatmig. Viele Folgen, positiv und negativ, werden erst nach deutlich längerer Nachbeobachtungszeit sichtbar. W.D. Ludwig und G. Schott von der Arzneimittelkommission der Deutschen Ärzteschaft (AkdÄ) thematisieren dieses Defizit in ihrem Artikel «Neue Arzneimittel in der Onkologie: Merkmale klinischer Zulassungsstudien und Argumente für die rasche Durchführung unabhängiger klinischer Studien nach der Zulassung» in diesem Sonderheft.

Allein in Deutschland leben heute 40000 Menschen, die eine bösartige Erkrankung im Kindes- und Jugendalter über- standen haben. In einer Leitlinien-Initiative streben die DGHO und die Gesellschaft für Pädiatrische Onkologie und Hämatologie $(\mathrm{GPOH})$ an, Kriterien für die Langzeitbeobachtung von Kindern und Erwachsenen nach einer medikamentösen Krebstherapie zu erarbeiten. Daten wurden in Deutschland vom Kinderkrebsregister und von den etablierten Studiengruppen der Erwachsenen erhoben. Auch das ist eine Studienkultur, die es zu erhalten gilt.

\section{Disclosure Statement}

Die Autoren haben für diese Arbeit keine Interessenkonflikte offenzulegen.

\section{Literatur}

1 Wörmann B, Wulf G, Hiddemann W: Klinische Studien in der Onkologie. Med Klein 1998;93:181189.

2 Lind J: A treatise of the scurvy in three parts. Containing an inquiry into the nature, causes and cure of that disease, together with a critical and chronological view of what has been published on the subject. London, A. Millar, 1753.

3 Bull JP: The historical development of clinical therapeutic trials. J Chron Dis 1959;10:218-248.

4 Medical Research Council: Clinical trial of patulin in the common cold. Lancet 1944;244:373-375

5 De Vita V: A history of cancer chemotherapy. Cancer Res 2008;68:8643-8653.
6 Frei E 3rd, Holland JF, Schneiderman MA, et al.: A comparative study of two regimens of combination chemotherapy in acute leukemia. Blood 1958; 13:1126-1148.

7 Fisher B, Carbone P, Economou SG, et al.: L-Phenylalanine mustard (L-PAM) in the management of primary breast cancer. N Engl J Med 1975;292:110122.

8 Bonadonna G, Brusamolino E, Valegussa P, et al.: Combination chemotherapy as an adjunct treatment in operable breast cancer. N Engl J Med 1976;294:405-410.

9 Vorschlag für eine Verordnung des Europäischen Parlaments und des Rates über klinische Prüfungen mit Humanarzneimitteln und zur Aufhebung der Richtlinie 2001/20/EG. Europäische Kommission 2012/0192, 2012.
10 Deutscher Bundestag: Gesetz zur Neuordnung des Arzneimittelmarktes in der gesetzlichen Krankenversicherung (Arzneimittelmarktneuordnungsgesetz - AMNOG). Bundesgesetzblatt 2010; Teil 1 (67):2262-2277.

11 Verordnung über die Nutzenbewertung von Arzneimitteln nach § 35a Absatz 1 SGB V für Erstattungsvereinbarungen nach $\S 130 \mathrm{~b}$ SGB V (ArzneimittelNutzenbewertungsverordnung - AM-NutzenV). Bundesgesetzblatt 2010; Teil 1(67):2324-2328.

12 Rimawi M, Hilsenbeck SG: Making sense of clinical trial data: Is inverse probability of censoring weighted analysis the answer to crossover bias? J Clin Oncol 2012;30:453-458. 